\title{
Skin Effect Aggregated Heating in RF MEMS Suspended Structures
}

\author{
Linda L.W. Chow* Zhongde Wang ${ }^{\dagger}$ Brian D. Jensen $^{\ddagger}$ \\ Kazuhiro Saitou* John L. Volakis ${ }^{\S}$ Katsuo Kurabayashi*† \\ *Dept. of Mechanical Engineering, University of Michigan, Ann Arbor, MI 48109, USA \\ ${ }^{\dagger}$ Dept. of Electrical Engineering and Computer Science, University of Michigan, Ann Arbor, MI 48109, USA \\ ${ }^{\ddagger}$ Dept. of Mechanical Engineering, Brigham Young University, Provo, UT 84602, USA \\ $\S$ Dept. of Electrical and Computer Engineering, Ohio State University, Columbus, OH 43210, USA \\ Email: katsuo@umich.edu
}

\begin{abstract}
This paper presents experimental data together with 2 modeling approaches to demonstrate the increased heating of MEMS suspended structures at radio frequencies due to skin effects. Distinguishable average temperature rises are measured at $2,13.5$, and $18 \mathrm{GHz}$ in a $616 \mu \mathrm{m} x$ $20 \mu \mathrm{m} \times 2.7 \mu \mathrm{m}$ suspended coplanar waveguide using 4-wire measurement configuration. Our measurements compare well with: (1) previous electromagnetic simulations and (2) a newly introduced analytical thermal model incorporating only skin effects. Buckling and plastic yielding have been observed during and after measurement. This study provides a simple and quantitative approach for the design of suspended structures such as low loss transmission lines, filters and switches with high power handling capability.

Index Terms-skin effect, microelectromechanical devices, transmission lines, coplanar waveguides, failure analysis.
\end{abstract}

\section{INTRODUCTION}

MEMS suspended structures within RF/microwave circuit components including transmission lines, switches and filters have exhibited very low loss without substratewave modes and dispersion [1], [2]. However, with limited thermal conduction paths, they suffer from low power handling capability owing to the localized heat generated in the metallic structures. Previous work demonstrated such localized temperature rise using modeling [3] and electro-opto-thermal probes [4]. However, the influence of the skin effect on such heating has not been explored.

This paper studies the impact of the skin effect behavior of RF MEMS. To carry out this study, we develop a simple and precise method to measure the average temperature rise in suspended MEMS structures using a 4wire measurement configuration [5] and RF power feeding. Unlike other micron-resolution temperature measurement techniques, such as infrared microscopy, scanning thermal microcopy, picosecond acoustics, time-domain thermoreflectance, and electro-optic probes [4], our method does not require sophisticated equipments and difficult calibration procedures.

Low power handling capability of MEMS suspended structures is often associated with low reliability due to thermally induced buckling, creep and even melting. When a suspended structure with its two ends anchored heats

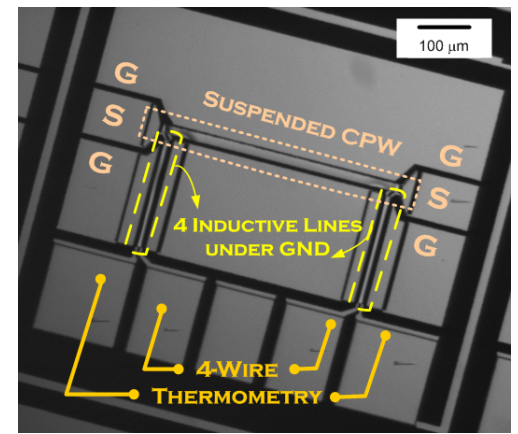

Fig. 1. Optical Image of a suspended CPW for average temperature measurement.

up and expands to the point of exceeding the structural stability limit, it undergoes unpredictable physical shape change, typically buckling upward. Buckling is the most catastrophic action, since it occurs at lower temperature as compared to creep and melting. Furthermore, the elastic limit is very low in metals ( $<0.5 \%$ rupture strain) and this leads to permanent buckling deformation (plastic yielding) even after cooling down.

An easy-to-use analytical thermal model incorporating the skin effect is also introduced, and compared with our previous electromagnetic (EM) simulations [6], [7] and experimental measurement. We are using this model to provide some useful guidelines for $\mathrm{RF} /$ microwave circuit components design using MEMS suspended structures in high power applications.

\section{Conceptual Design \& Sensing Mechanism}

Among various types of transmission lines, coplanar waveguides $(\mathrm{CPW})$ are more readily fabricated without adding inductances at the RF grounding. A $50 \Omega \mathrm{CPW}$ incorporated into a suspended beam with a 4-wire temperature measurement configuration is designed in this study.

The basic mechanism for this temperature measurement technique over the suspended CPW is as follows: With RF power passing through, the suspended CPW heats up, resulting in an increase of the electrical resistivity. Our 4-wire measurement configuration implemented by 


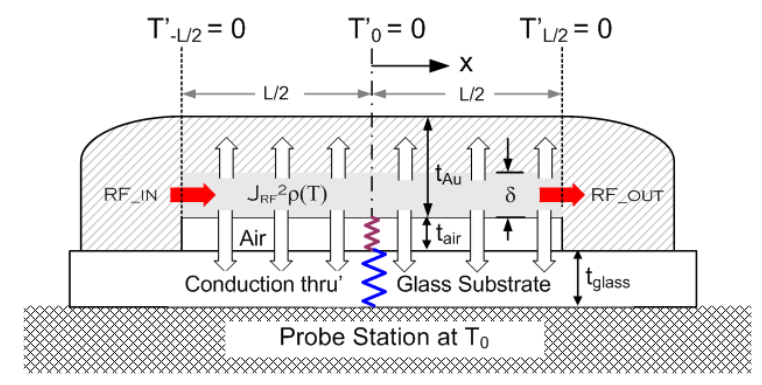

Fig. 2. Schematic diagram for our proposed analytical thermal model

adding four extra probe pads on the two ends of the suspended CPW via four on-chip inductive transmission lines is shown in Fig. 1. The suspended CPW is fed with a small DC current and the corresponding DC voltage is measured. The average temperature rise of the entire suspended CPW is then obtained. It is important to note that DC current adds negligible power towards temperature rise $(<1 \%)$.

\section{Modeling APPROACHES}

\section{A. EM EFE-BI coupled Thermal FEM Simulation}

To accurately account for the electrical loss in the structure, a full-wave EM simulation using EFE-BI with a preconditioner [7] has been carried out. To capture the current crowding at the edges along the CPW length (the "edge effects"), the heat conduction equation is then solved via a $2 \mathrm{D}$ FEM and by using the EFE-BI results as heat source.

\section{B. Analytical Thermal Model Capturing only Skin Effects}

Owing to the small MEMS dimensions $(<0.001$ wavelength), we assume that the edge effects and EM radiation losses are minimal as compared to skin effects. As such, we proceed to develop an analytical thermal model that only captures the skin effects, as in Fig. 2.

In addition, since radio frequencies $(\sim 1 \mathrm{~ns})$ are much higher than heat diffusion time $(\sim 1 \mu \mathrm{s})$, a steady rms value of RF power is used rather than a time-varying value. Specifically, we model the temperature rise in steady state, as our data-logging in experiment is designed to be longer than a minute.

The 1D heat conduction governing equation with air conduction through the air gap and the glass substrate, and the accompanying boundary conditions are:

$$
\begin{gathered}
t_{A u} \nabla \cdot k_{A u} \nabla T+J_{R F}^{2} \rho=\left(\frac{k_{\text {glass }}}{t_{\text {glass }}}+\frac{k_{\text {air }}}{t_{\text {air }}}\right)\left(T-T_{0}\right) \\
\left.\frac{\partial T}{\partial x}\right|_{x=0}=0,\left.\frac{\partial T}{\partial x}\right|_{x=\frac{L}{2}}=0
\end{gathered}
$$

To obtain a closed form solution, the skin depth and CPW length are assumed constant with temperature. This assumption allows us to re-write the left side of (1) as:

$$
k_{A u} t_{A u} \nabla^{2} T+J_{R F}^{2} \delta \rho_{0}\left[1+\alpha_{t}\left(T-T_{0}\right)\right]
$$

The temperature rise with reference to the ambient temperature is then solved by (1):

$$
\begin{gathered}
\Delta T=T-T_{0}=\frac{\beta}{\gamma^{2}}\left[\frac{1}{\gamma} \sinh (\gamma x)+\frac{1-\cosh \left(\frac{\gamma L}{2}\right)}{\sinh \left(\frac{\gamma L}{2}\right)} \cosh (\gamma x)-1\right] \\
\gamma=\sqrt{\frac{1}{k_{A u}}\left|\left(\frac{k_{\text {glass }}}{t_{\text {glass }}}+\frac{k_{\text {air }}}{t_{\text {air }}}\right)-\frac{\alpha_{t} J_{R F}^{2} \delta \rho_{0}}{t_{A u}}\right|} \\
\beta=\frac{J_{R F}^{2} \delta \rho_{0}}{k_{A u} t_{A u}} \quad J_{R F}=\frac{1}{w \delta} \sqrt{\frac{P_{R F}}{Z_{0}}}
\end{gathered}
$$

where $\mathrm{k}$ and $\mathrm{t}$ are the thermal conductivity and thickness respectively. We note that the peak temperature rise is at the mid-length:

$$
\left.\triangle T\right|_{x=0}=\frac{\beta}{\gamma^{2}}\left[\frac{1-\cosh \left(\frac{\gamma L}{2}\right)}{\sinh \left(\frac{\gamma L}{2}\right)}-1\right]
$$

and the average temperature rise is given by

$$
\triangle T_{\text {avg }}=\frac{2 \beta}{\gamma^{4} L}\left[\left(\cosh \left(\gamma \frac{L}{2}\right)-1\right)(1-\gamma)-\frac{\gamma L}{2}\right]
$$

\section{EXPERIMENTS}

\section{A. Fabrication}

Many RF MEMS components are made of gold to achieve low resistivity and inertness to oxidation. Nonetheless, this temperature measurement technique can be applicable to any electrically conductive material. A $30 \mathrm{~nm}$ $\mathrm{Cr} / 1 \mu \mathrm{m} \mathrm{Au}$ thin film was initially sputtered on a glass substrate and lithographically patterned as the probe pads and 4 inductive lines. A photoresist sacrificial layer was then spun on to define the anchor, and $2.7 \mu \mathrm{m}$ Au thin film was sputtered as the suspended signal line of the CPW, and the suspended ground connection above the 4 inductive lines. After the suspended structures were patterned and wet-etched, die-sawing, sacrificial layer removal and finally $\mathrm{CO}_{2}$ critical dry were performed.

\section{B. Setup and Calibration}

The experimental setup in Fig. 3 was calibrated using the network analyzer (HP8517B S-parameter test set) with and without adding DC probes from 0.5 to $20 \mathrm{GHz}$. Nonsuspended CPWs were first measured to characterize the power loss either in the substrate or due to radiation, which have been found negligibly small $\left(<0.1 \mathrm{~dB}\right.$ in $\left.\mathrm{S}_{21}\right)$. Subsequently, we measured the suspended CPW and three frequencies were chosen with good impedance match for temperature-power measurement.

To maximize the thermal sensitivity, the suspended $\mathrm{CPW}$ was tested over a range of RF power from low to very high up to the equipment specifications. To do so, the RF power from a RF synthesized sweeper (HP 8364A) was 


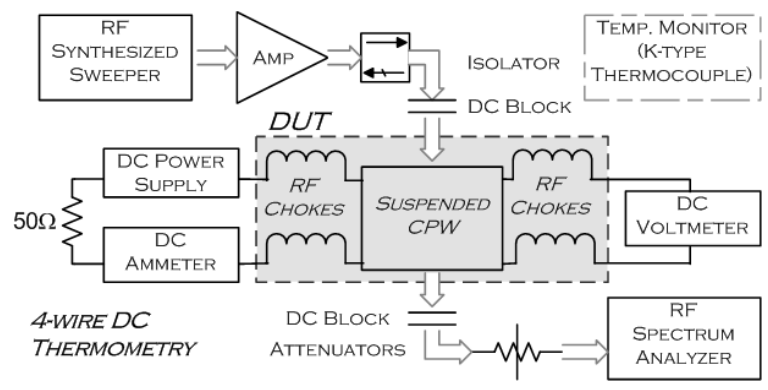

Fig. 3. Schematic setup diagram and RF power-temperature measurement

fed into a power amplifier (Hughes Research: K-band and X-band Traveling Wave Tubes, Miteq: 1-2GHz Amplifier) to the suspended CPW through an isolator and DC blocks, and was monitored using a spectrum analyzer (HP 8562A). The 4-wire thermometric DC circuitry was connected to our suspended CPW via four on-chip RF inductive lines as shown in Fig. 1. A $50 \mathrm{~mA}$ DC current was generated from a DC Power Supply (HP E3630A), corresponding to $<1 \%$ in temperature rise. The DC voltage was then measured by the 6.5-digit multi-meter (HP 34401A). The ambient temperature was also monitored using a K-type thermocouple with a $0.5^{\circ} \mathrm{C}$ accuracy rating.

\section{Temperature and RF Power Extraction}

Heating of the suspended beam causes its resistance to rise, resulting in a $\mathrm{DC}$ voltage difference, $\mathrm{V}_{D C}$, across the beam of

$$
V_{D C}=I_{D C} R=I_{D C} \frac{L}{w t} \rho_{0}\left(1+\alpha_{t} \triangle T_{a v g}\right)
$$

Dimensions such as thickness, $t$, width, w, and length, $\mathrm{L}$ were precisely measured with a surface profilometer (Dektak 6M) and an interferometer (Zygo Newview 5000). The temperature coefficient of resistivity, $\alpha_{t}$, and the resistivity at ambient temperature, $\rho_{0}$, were calibrated using a van der Pauw structure heated by a temperature controlled thermal chuck.

The RF power was obtained from the calibration of cable/connector/DC probes. Resistance rises from 0.5 to $0.6 \Omega$ and $<4 \%$ of carried RF power is absorbed as heating, hence, there is insignificant change in the Sparameters with different carried RF power levels. Half of the power loss is assumed to be symmetrically dissipated on the DC circuit, which might contribute to some uncertainties on RF power input into our suspended $\mathrm{CPW}$. However, the measured temperature is within $2 \%$ of experimental error.

\section{RESUlts AND Discussions}

\section{A. Modeling Prediction}

The closed form solution in Equation (3) is used as a starting point with an iterative code to account for temper-

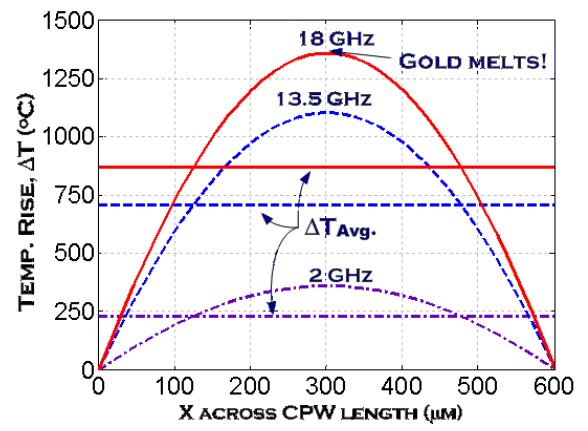

Fig. 4. "Parabolic" shape temperature rise distribution, $\triangle T$, along the suspended CPW is modeled using EFE-BI in EM and FEM in thermal simulation; horizontal lines are the corresponding average temperature rises, $\triangle \mathrm{T}_{\text {avg }}$

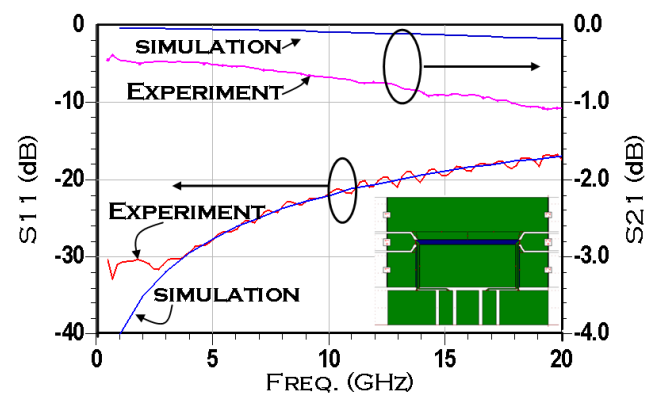

Fig. 5. Measured and simulated isolation and insertion loss of our suspended CPW

ature dependent skin depth and suspended CPW length. A $0.5 \mathrm{~W}$ RF power input results in a peak temperature rise (average temperature rise) of $82{ }^{\circ} \mathrm{C}\left(60{ }^{\circ} \mathrm{C}\right)$ at $18 \mathrm{GHz}$, $72{ }^{\circ} \mathrm{C}\left(50{ }^{\circ} \mathrm{C}\right)$ at $13.5 \mathrm{GHz}$, and $26^{\circ} \mathrm{C}\left(22^{\circ} \mathrm{C}\right)$ at $2 \mathrm{GHz}$, which are very close to the full wave $\mathrm{EM}$ simulation results: $85{ }^{\circ} \mathrm{C}\left(55{ }^{\circ} \mathrm{C}\right)$ at $18 \mathrm{GHz}, 73{ }^{\circ} \mathrm{C}\left(47{ }^{\circ} \mathrm{C}\right)$ at $13.5 \mathrm{GHz}$, and $27{ }^{\circ} \mathrm{C}\left(18{ }^{\circ} \mathrm{C}\right)$ at $2 \mathrm{GHz}$. As the results, we can conclude that skin effect is predominant in MEMS scale and radio frequencies. Interestingly, as in Fig. 4, a $5 \mathrm{~W}$ RF power input of $18 \mathrm{GHz}$ can reach $1300{ }^{\circ} \mathrm{C}$ causing melting of the gold structure!

\section{B. Isolation and Insertion Loss of Suspended CPW With and Without DC Probes}

The S-parameters of our suspended CPW match well with the simulation using IE3D [8] as shown in Fig. 5. However, the measurement shows substantial deviation after the DC probes are attached. We did observe that as long as the DC probes were in place, S-parameters showed no change when the DC measurement equipments were either connected or disconnected. Hence, we believe that the bypass capacitors in the DC probes were responsible for creating resonances, resulting in degradation of the RF performance of the suspended beam. As mentioned, we 


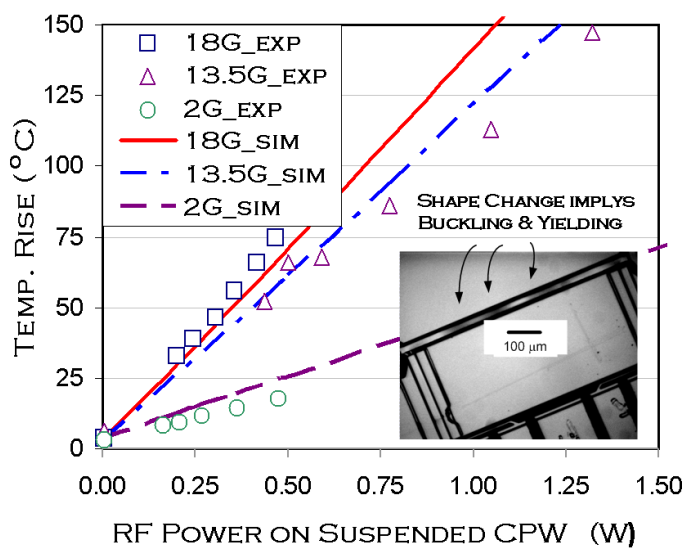

Fig. 6. Heating in the suspended beam due to skin effects, and (inset figure) buckling and yielding observed during and after RF power measurement.

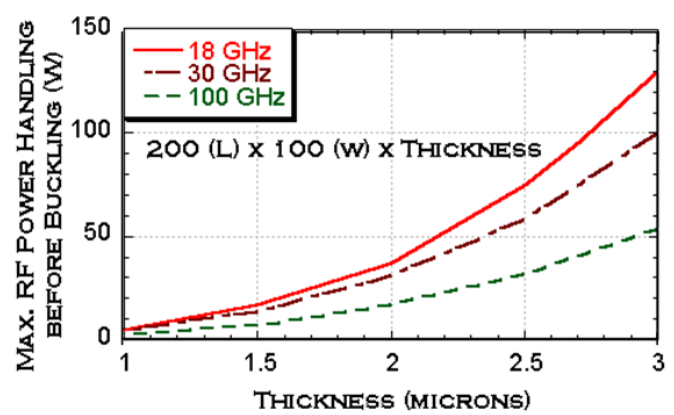

Fig. 7. RF Power handling enhanced by thick structures in 18,30 and $100 \mathrm{GHz}$

picked three impedances well matched frequencies: $2,13.5$ and $18 \mathrm{GHz}$ for the power measurement.

\section{Average Temperature Measurement with various $R F$ Frequencies and RF Power Levels}

Three distinct curves of average temperature rise for the $616 \mu \mathrm{m} \times 20 \mu \mathrm{m} \times 2.7 \mu \mathrm{m}$ suspended CPW are displayed in Fig. 6 at 3 radio frequencies. $0.5 \mathrm{~W}$ RF power can have an average temperature rise of $\sim 75{ }^{\circ} \mathrm{C}$ at $18 \mathrm{GHz}, \sim 60{ }^{\circ} \mathrm{C}$ at $13.5 \mathrm{GHz}$, and $\sim 20{ }^{\circ} \mathrm{C}$ at $2 \mathrm{GHz}$. Measurements show good agreement with the analytical thermal model, implying that the skin effect is the key parameter attributing to suspended MEMS heating at RF frequencies.

\section{Power Handling Enhanced by Thick Structure}

Buckling and plastic yielding have been observed during and after removal of the measurement equipment as the inset of Fig. 6. Our suggested analytical thermal model predicts that a stress-free $616 \mu \mathrm{m} \times 20 \mu \mathrm{m} \times 2.7 \mu \mathrm{m}$ suspended beam starts to buckle, corresponding to an $18 \mathrm{GHz} \mathrm{RF}$ power input of only $48 \mathrm{~mW}$ ! In practice, many suspended CPWs are short and wide; however, even a $200 \mu \mathrm{m} \times 100 \mu \mathrm{m} \times 1 \mu \mathrm{m}$ suspended beam can handle only $5 \mathrm{~W} \mathrm{RF}$ power at $18 \mathrm{GHz}, 4 \mathrm{~W}$ at $30 \mathrm{GHz}$, and $2 \mathrm{~W}$ at $100 \mathrm{GHz}$. Fig. 7 shows how RF power handling capability can be effectively enhanced by a thicker structure. Increasing the thickness from $1 \mu \mathrm{m}$ to $3 \mu \mathrm{m}$ can deliver $130 \mathrm{~W}$ at $18 \mathrm{GHz}, 100 \mathrm{~W}$ at $30 \mathrm{GHz}$ and $54 \mathrm{~W}$ at $100 \mathrm{GHz}$, which leads to a 25 -fold power handling!

\section{CONCLUSION}

The focus of this paper is to experimentally examine skin effect heating in MEMS suspended structures. The experimental data is also experimentally validated with a hybrid finite element- boundary integral method coupled with thermal simulation. Our results have indicated that heating in suspended MEMS structures is a strong function of frequency and gives rise to thermal-stress-induced structural instability and permanent shape change. This limits their reliability and power handling capability. The model predictions show good agreement with the experimental data and allow for guidelines to be used in RF MEMS design. For example, an increase of the MEMS structure thickness by a few microns can substantially decrease temperature rise and thus permits the suspended CPWs to transmit more than 25 times RF power.

\section{ACKNOWLEDGMENT}

This work was supported by the National Science Foundation under contracts nos. ECS-0115222 and ECS0330963. The authors would like to thank Bryan J.J. Hung and Prof. Gabriel M. Rebeiz for helpful assistance and fruitful discussion in RF power experiment at Radiation Lab, University of Michigan, Ann Arbor.

\section{REFERENCES}

[1] Y. Yoshida, T. Nishino, J. Jiao, S.-S. Lee, Y. Suehiro, K. Miyaguchi, T. Fukami, M. Kimata,O. Ishida, "A grounded coplanar waveguide with a metallized silicon cavity fabricated by front-surface-only processes," Sensors and Actuators, A: Physical, vol. 111, no. 1, pp. 129-134, 2004.

[2] K.J. Herrick, T.A. Schwarz, and L.P.B. Katehi, "Si-Micromachined coplanar waveguides for use in high-frequency circuits," IEEE Trans. Microwave Theory \& Tech., vol. 46, no. 6, pp. 762-768, 1998.

[3] J.B. Rizk, E. Chaiban, and G.M. Rebeiz, "Steady state thermal analysis and high-power reliability considerations of RF MEMS capacitive switches," IEEE MTT-S Int. Microwave Symp. Dig., vol. 1, pp. 239-43, 2002.

[4] R.M. Reano, D. Peroulis, J.F. Whitaker, and L.P.B. Katehi, "Electro/thermal measurements of RF MEMS capacitive switches", IEEE MTT-S Int. Microwave Symp. Dig., vol. 3, pp. 1923-1926, 2003.

[5] J. Semancik, "4-wire measurement," Sensors, vol. 18, no. 5, pp. 4450,2001

[6] B.D. Jensen, K. Saitou, J.L. Volakis, and K. Kurabayashi, "Fully Integrated Electrothermal Multi-Domain Modeling of RF MEMS Switches,"IEEE Microwave and Wireless Components Letters, vol. 13, no. 9, pp. 364-366, 2003.

[7] Z. Wang, B.D. Jensen, J.L. Volakis, K. Saitou, and K. Kurabayashi, "Integrated Electrothermal Analysis of RF-MEMS Switches by Using Extended Finite Element-Boundary Integration Method," submitted to IEEE J. Microelectromech. Syst.

[8] http://www.zeland.com 